\title{
Engineered andrographolide nanosystems for smart recovery in hepatotoxic conditions
}

\author{
This article was published in the following Dove Press journal: \\ International Journal of Nanomedicine \\ 9 October 2014 \\ Number of times this article has been viewed
}

\author{
Partha Roy ${ }^{1,2}$ \\ Suvadra Das' \\ Runa Ghosh Auddy ${ }^{1,3}$ \\ Arup Mukherjee ${ }^{1,3}$ \\ 'Division of Pharmaceutical and Fine \\ Chemicals Technology, Department \\ of Chemical Technology, University \\ of Calcutta, Kolkata, India; ${ }^{2}$ Faculty \\ of Technology (Pharmaceutical), \\ Universiti Malaysia, Pahang, Malaysia; \\ ${ }^{3}$ Centre for Research in Nanoscience \\ and Nanotechnology, University \\ of Calcutta, Kolkata, India
}

\begin{abstract}
Andrographolide (AG) is one of the most potent labdane diterpenoid-type free radical scavengers available from plant sources. The compound is the principal bioactive component in Andrographis paniculata leaf extracts, and is responsible for anti-inflammatory, anticancer, and immunomodulatory activity. The application of AG in therapeutics, however, is severely constrained, due to its low aqueous solubility, short biological half-life, and poor cellular permeability. Engineered nanoparticles in biodegradable polymer systems were therefore conceived as one solution to aid in further drug-like applications of AG. In this study, a cationic modified poly(lactic-co-glycolic) acid nanosystem was applied for evaluation against experimental mouse hepatotoxic conditions. Biopolymeric nanoparticles of hydrodynamic size of $229.7 \pm 17.17 \mathrm{~nm}$ and $\zeta$-potential $+34.4 \pm 1.87 \mathrm{mV}$ facilitated marked restoration in liver functions and oxidative stress markers. Superior dissolution for bioactive AG, hepatic residence, and favorable cytokine regulation in the liver tissues are some of the factors responsible for the newer nanosystemassisted rapid recovery.
\end{abstract}

Keywords: andrographolide, engineered nanosystems, poly(lactic-co-glycolic) acid, cytokine regulation, hepatotoxicity

\section{Introduction}

Progressive liver damage is one of the most serious health hazards afflicting a growing population across the globe. Hepatic injury is one of the top ten recorded causes of death in the US. ${ }^{1}$ The liver is the site for drug detoxification and biotransformation of exogenous chemicals that penetrate body systems via different routes. Therapeutic strategies to alleviate conditions of hepatic injury and inflammation of the liver are however very rare. Furthermore, as only a few therapeutic choices are available, these lack hugely in specificity. ${ }^{2}$ Agents like antiviral drugs, steroids, and vaccines are particularly harmful to the liver when applied in prolonged treatment conditions, such as antitubercular, antiretroviral, or anticancer chemotherapy.,

Natural products and traditional herbal medicines are currently attracting a renewed focus as safer and effective alternatives in hepatorestorative therapy. Select supplements of herbal origin are some of the confirmed hepatoprotectants, but their applications cannot be approved because of the inherent multichemical nature of plant drugs and lack of pinpoint activity-proficiency proof. ${ }^{5}$ Andrographolide (AG) is one of the most powerful anti-inflammatory agents available directly from plant sources. The compound effectively reduces production of reactive oxygen species (ROS), TNF $\alpha$, nitrous oxide species, and prostaglandin $\mathrm{E}_{2}$ in vitro. ${ }^{6}$ Aqueous extract of the Andrographis paniculata (family: Acanthaceae) plant was reported to have anti-inflammatory, anticancer, hepatoprotective, and immunomodulatory activities. ${ }^{7-9}$ The plant is well known as the "king of bitters", and is applied in traditional Indian and Chinese systems of
Correspondence: Arup Mukherjee Division of Pharmaceutical and Fine Chemicals Technology, Department of Chemical Technology, University of Calcutta, 92 APC Road, Kolkata 700009 , India

$\mathrm{Tel}+913323508387$

Fax +9| 3323519755

Email arupm1234@gmail.com 
medicine in order to alleviate chronic digestive disorders and liver-centric diseases. Early attempts with AG and synthetic prodrugs against arthritis and leishmaniasis were aborted, due to constraints in dissolution and targetability. ${ }^{10}$ The significant clinical potential of AG was thus marred due to poor aqueous solubility and short biological half-life of only 2 hours. ${ }^{11}$ Rapid metabolic sulfonation in the duodenum-jejunum areas and P-glycoprotein-efflux activities are some of the root reasons identified recently for poor bioactivity of similar terpenoids. ${ }^{12}$ Some newer semisynthetic AG derivatives were very recently reported for solubility enhancement and for likely efficacy in hepatoprotection and retardation of apoptosis. ${ }^{13}$ We were further successful in applying different nanoparticle (NP)engineering techniques for favorable liver-localized biological AG activity. ${ }^{14}$ At least one report was recently noticed on the synthesis of solid-lipid NPs for pharmacokinetic enhancement and antihyperlipidemic activity of AG. ${ }^{15}$

Progressive oxidative stress is one ubiquitous health hazard, and carbon tetrachloride $\left(\mathrm{CCl}_{4}\right)$ liver damage in rodents is an established in vivo model for similar evaluations. $\mathrm{CCl}_{4}$ is known to cause deoxyribonucleic acid methylation and free radical-mediated hepatocellular damages. ${ }^{16}$ When metabolized by the cytochrome P450 (CYP2E1) enzyme system, $\mathrm{CCl}_{4}$ forms trichloromethyl $\left(\cdot \mathrm{CCl}_{3}\right)$ and trichloroperoxyl $\left(\cdot \mathrm{OOCCl}_{3}\right)$ radicals. Both species initiate a free radical-mediated lipid peroxidation that culminates in degeneration of membrane lipids and liver damage. The compound educes a marked rise in hepatic marker enzymes and lipid-peroxidation products like malonyldialdehyde (MDA) in the mouse. ${ }^{17}$ The aim of this study was to explore drug-like hepatoprotective activity against $\mathrm{CCl}_{4}$ acute liver damage models for new AG nanosystems and AG alone extracted and purified directly from native A. paniculata leaves. The principles of NP engineering were applied in order to develop cationic modified AG in biopolymeric nanosystems for a size-directed bioactive dissolution and hepatorestorative evaluations upon peroral administration.

\section{Materials and methods Materials}

The biopolymers poly(lactic-co-glycolic) acid (PLGA; 50:50, molecular weight [MW]40,000-75,000), chitosan(Cs; medium MW), Pluronic ${ }^{\circledR}$ F-127 (MW 12,600), and dialysis tubing (MW cutoff 12,400) were all purchased from Sigma-Aldrich Co. (St Louis, MO, USA). Solvents and water used were of high-performance liquid chromatography (HPLC) grade, and were procured from Merck KGaA (Darmstadt, Germany). Biochemical analysis kits were from Merck KGaA and eBioscience (San Diego, CA, USA). Analysis procedures followed were as prescribed by the manufacturer unless mentioned otherwise. A. paniculata whole plant and the leaves were collected at the end of September 2012 from the medicinal plant gardens of Ramkrishna Mission, Narendrapur, Kolkata, India. The plant specimens were identified and authenticated by comparison, with reference to the herbarium (CNH/68/2012/Tech.II/887), preserved at the Indian Botanical Gardens, Central Herbarium, Howrah, India.

\section{Extraction, purification, and characterization}

Powdered A. paniculata leaves (500 g) were extracted in $95 \% \mathrm{v} / \mathrm{v}$ aqueous ethanol in a Soxhlet apparatus under refluxing for 10 hours. The extract was concentrated and subjected to two cycles of solid-liquid extraction in $100 \mathrm{~mL}$ of $n$-hexane:acetone $(85: 15)$ mixture to eliminate any residual chlorophyll. The resultant solid was dissolved in $250 \mathrm{~mL}$ ethanol under refluxing, and cooled, filtered, and mixed with activated charcoal for hot filtration under suction. The light-yellow liquid was concentrated to $25 \mathrm{~mL}$ under reduced pressure and charged over a 160-mesh activated neutral alumina column $(80 \times 6 \mathrm{~cm})$, and AG was finally eluted against $80 \% \mathrm{v} / \mathrm{v}$ aqueous ethanol. The diterpenoid AG content was confirmed in an ultraviolet (UV)-visible spectrophotometer (UV-2550; Shimadzu, Kyoto, Japan). The colorless eluent was concentrated by evaporation and crystallized under cooling in a refrigerator. Purified AG was finally recrystallized from absolute ethanol under anhydrous conditions and dried in a vacuum oven at $60^{\circ} \mathrm{C}$ for preservation.

A dilute solution of $\mathrm{AG}$ in chloroform was analyzed in a UV-visible spectrophotometer, and the maximum absorbance $\left(\lambda_{\max }\right)$ values were recorded. Fourier-transform infrared (FTIR) experiments were carried out with an FTIR 670 Plus (Jasco, Easton, MD, USA) and the melting point of the extracted product was determined with melting-point apparatus (Systonic, Panchkula, Haryana, India). Percentage $C$, $\mathrm{H}$, and $\mathrm{N}$ composition of $\mathrm{AG}$ was recorded in a CHNS analyzer model CHNS-932 (Leco Corporation, St Joseph, MI, USA). Mass spectra of purified AG extracted were obtained in a Q-Tof micro-mass spectrometer (Waters Corporation, Milford, MA, USA). Reverse-phase HPLC (RP-HPLC) analysis for AG was carried out following isocratic elution using a mobile phase of acetonitrile: $0.1 \%(\mathrm{v} / \mathrm{v})$ phosphoric acid in water $(40: 60, \mathrm{v} / \mathrm{v})$ at a flow rate of $1 \mathrm{~mL} / \mathrm{minute}$ in a $_{18}$ column $(25 \times 4.6 \mathrm{~mm}, 5 \mu \mathrm{m}$; Supelco, Bellefonte, PA, USA) attached with one HPLC system (Waters Corporation). A peak area $(y)$ versus concentration $(x)$ graph for AG was recorded, and the linearity equation developed was applied to estimate AG concentrations throughout. ${ }^{18}$ 


\section{Engineered nanosystem design}

A facile emulsion solvent-evaporation technique was adopted for the synthesis of AG nanosystems in PLGA. Cationic modification was achieved by polyelectrolyte deposition of Cs biopolymer on AG in PLGA nanosystems. Briefly, $50 \mathrm{mg}$ PLGA and $2.5 \mathrm{mg}$ of AG in $3 \mathrm{~mL}$ of chloroform was emulsified in $12 \mathrm{~mL}$ of $1 \% \mathrm{w} / \mathrm{v}$ aqueous Pluronic F-127 solution by sonication (Sonics, Milpitas, CA, USA) for 1 minute and homogenization (Omni International, Kennesaw, GA, USA) at 20,000 rpm for 20 minutes. AG nanoparticles (AGNPs) formed were harvested by ultracentrifugation at $30,000 \mathrm{rpm}$ for 25 minutes at $4^{\circ} \mathrm{C}$. An aqueous dispersion of AGNPs $(2 \mathrm{~mL}$, $0.3 \% \mathrm{w} / \mathrm{v})$ was added dropwise to the Cs solution $(0.3 \% \mathrm{w} / \mathrm{v})$ in aqueous acetic acid $(1 \% \mathrm{v} / \mathrm{v})$ under magnetic stirring at $10^{\circ} \mathrm{C}$ for 1 hour. Cs-modified AGNPs (CsAGNPs) were further harvested similarly by centrifugation, and were stored at $4^{\circ} \mathrm{C}$ until further analysis. Fluorescence-labeled PLGA NPs were prepared separately following the same procedure, but by replacing AG with fluorescein isothiocyanate. Each preparation was done in quadruplicate for drug-loading, $\zeta$-analysis, and release studies. Final AG nanosystems were similarly received by ultracentrifugation at $30,000 \mathrm{rpm}$ for 25 minutes at $4^{\circ} \mathrm{C}$.

\section{Characterization for engineered nanosystems}

Particle characterization was carried out by systematic evaluation of particle size, polydispersity index, $\zeta$-potential, and surface morphology. Particle size, polydispersity index, and $\zeta$-potential were all measured in a Zetasizer Nano ZS (Malvern Instruments, Malvern, UK), while particle morphology was studied with atomic force microscopy (AFM; NanoScope 3A; Veeco Instruments, Inc., Plainview, NY, USA) and transmission electron microscopy (TEM; JEM 2100; JEOL, Japan). AFM tracing was done in tapping mode using an RTESP tip with $267-328 \mathrm{kHz}$ resonance frequency at a scan speed of $1.2 \mathrm{~Hz}$. For TEM measurements, $10 \mu \mathrm{L}$ of CsAGNP suspension in water were carefully placed on a 300-mesh formvarcoated copper TEM grid (Ted Pella, Inc., Redding, CA, USA) and stained in $2 \% \mathrm{w} / \mathrm{v}$ of uranyl acetate solution for 1 minute. The excess solution on the grid was removed using a piece of fine-filter paper, and the samples were allowed to air-dry for 10 hours prior to imaging the particles under TEM. AGNPentrapment efficiency was recorded in HPLC as described previously. The mass of AG in solution before and after final NP harvesting in the supernatant was estimated in HPLC for determination of molecular mass load. ${ }^{19}$

The X-ray diffraction (XRD) patterns of AG extracted and the new nanosystem CsAGNPs were obtained in an X-ray diffractometer (PANalytical, Almelo, the Netherlands) at an applied voltage of $40 \mathrm{kV}$. The scanned angle was set from $3^{\circ} \leq 2 \theta \geq 50^{\circ}$, and the scanned rate was $2 \mathrm{~min}^{-1}$. For FTIR functional group analysis, $\mathrm{KBr}$ disks impregnated with NPs were scanned against blank over a wave number range of 4,000-400 $\mathrm{cm}^{-1}$. Cs content in the CsAGNP nanosystem was determined quantitatively following electrostatic interaction reactions of Cs with alizarin red dye before and after NP harvesting in the supernatant. ${ }^{19}$

\section{In vitro dissolution}

CsAGNPs equivalent to $2 \mathrm{mg}$ AG were dispersed separately in $1 \mathrm{~mL}$ of $100 \mathrm{mM}$ phosphate-buffer solution and washed completely into dialysis bags for equilibrating dissolution analysis. A buffer of $\mathrm{pH} 7.4$ was used throughout. ${ }^{20}$ The dialysis bags were placed in individual glass vials containing $10 \mathrm{~mL}$ of phosphate buffer maintained at $37^{\circ} \mathrm{C}$ over a magnetic stirrer, and $\mathrm{AG}$ dissolution over time was recorded in HPLC analysis. The buffer content in the dissolution vials was substituted with fresh buffer medium at each time interval in order to maintain sink conditions. The release data were averaged from four different experiments in each case, and were further fitted in Korsmeyer-Peppas models for iterative release exponent analysis using SigmaPlot ${ }^{\circledR} 6.0$ software (Systat Software, Inc., San Jose, CA, USA).

\section{In vivo evaluation of engineered nanosystems}

\section{Animals and treatments}

Healthy male Swiss albino mice (body weight $25 \pm 2 \mathrm{~g}$ ) were used for the study. Animals were housed in plastic cages bedded with rice husk, and were allowed to adapt for 5 days in the laboratory conditions of $25^{\circ} \mathrm{C} \pm 2^{\circ} \mathrm{C}$ and $12 / 12$ light/dark cycles. All animals received humane care, and the experiments were conducted as per the approval of the Institutional Animal Ethics Committee, Government of India (506/05/b/CPCSEA). They were given free access to a standard pellet diet (Hindustan Unilever Limited, Mumbai, India) and water ad libitum. The animals were fasted overnight, and on the day of the experiment only water was allowed. The experiment involved four groups of six animals each in the following treatment schedule:

- Group I: control animals gavaged with saline $10 \mathrm{~mL} / \mathrm{kg}$ body weight for 7 days

- Group II: saline $+\mathrm{CCl}_{4}, 10 \mathrm{~mL} / \mathrm{kg}$ body weight in $0.1 \%$ w/v olive oil, intraperitoneally

- Group III: powdered AG in saline $50 \mathrm{mg} / \mathrm{kg}$ body weight gavaged $+\mathrm{CCl}_{4}, 10 \mathrm{~mL} / \mathrm{kg}$ body weight in $0.1 \% \mathrm{w} / \mathrm{v}$ olive oil, intraperitoneally 
- Group IV: CsAGNPs in saline equivalent to AG $50 \mathrm{mg} / \mathrm{kg}$ body weight gavaged $+\mathrm{CCl}_{4} 10 \mathrm{~mL} / \mathrm{kg}$ body weight in $0.1 \% \mathrm{w} / \mathrm{v}$ olive oil, intraperitoneally.

Groups III and IV received the respective daily treatment of AG or CsAGNPs for the entire 7-day study period. A single dose of $\mathrm{CCl}_{4}$ was administered on day 7 (groups II, III, and IV) 1 hour after the final treatment to induce hepatotoxicity. ${ }^{21}$ Group I served as the normal control while group II was the $\mathrm{CCl}_{4}$ hepatotoxic control group.

\section{Measurement of liver enzymes in serum}

Twenty-four hours after the $\mathrm{CCl}_{4}$ injection, the animals were anesthetized and the blood was collected by cardiac puncture. Collected blood samples were centrifuged $(3,500 \mathrm{rpm} / \mathrm{min}$, 10 minutes at $\left.4{ }^{\circ} \mathrm{C}\right)$ and the serum separated and stored at $-20^{\circ} \mathrm{C}$ for subsequent determination of biochemical parameters. The activities of aspartate aminotransferase (AST), alanine transaminase (ALT), and alkaline phosphatase (ALP) were determined using standard analysis kits (Ecoline; Merck KGaA).

\section{Tissue samples}

Animals were killed by cervical dislocation immediately after collection of blood, and the liver tissue in each case was dissected out. Liver samples were washed with ice-cold saline and stored in $\mathrm{a}-20^{\circ} \mathrm{C}$ freezer for further analysis.

\section{Histopathology}

For histopathological analysis, the liver tissues from two animals in each group were fixed in $10 \%$ formalin, processed following routine histology procedures, and embedded in paraffin. Tissue sections $(6-7 \mu \mathrm{m})$ were then stained with hematoxylin and eosin dye, and subjected to photomicroscopy (B1 series; Motic, Xiamen, People's Republic of China) observations. ${ }^{22}$

\section{Preparation of liver-tissue homogenate}

Individual liver tissue in all other cases was crushed in a tissue homogenizer, and $10 \% \mathrm{w} / \mathrm{v}$ tissue homogenates were prepared with cold $50 \mathrm{mM}$ sodium phosphate buffer $(\mathrm{pH} 7.0)$ containing $0.1 \mathrm{mM}$ ethylenediaminetetraacetic acid. Solutions were centrifuged at $6,000 \times g$ at $4^{\circ} \mathrm{C}$ for 20 minutes, and the supernatant was collected for different biochemical analyses.

\section{Determination of lipid peroxidation}

The extent of lipid peroxidation was estimated spectrophotometrically in the tissue homogenates following estimation of MDA in the thiobarbituric acid reaction, ${ }^{23}$ and was expressed in terms of $\mu \mathrm{M} / \mathrm{mg}$ of liver tissue.

\section{Determination of antioxidant-enzyme markers}

Superoxide dismutase (SOD; EC1.15.1.1) in the liver was assayed at $480 \mathrm{~nm}$ based on the inhibitory effect on epinephrine autoxidation to adrenochrome in an alkaline medium (pH 10.2), ${ }^{24}$ and the catalase (CAT; EC 1.11.1.6) activity was measured at $240 \mathrm{~nm}$ from the rate of decomposition reactions of $\mathrm{H}_{2} \mathrm{O}_{2} \cdot{ }^{25}$ Glutathione (GSH) peroxidase (GPx) activity was determined by its ability to catalyze the oxidation of GSH by tert-butyl hydroperoxide. ${ }^{26}$ GSH reductase (GR) was measured by the decrease in absorbance due to the oxidation of nicotinamide adenine dinucleotide phosphate (NADPH) to reduced nicotinamide adenine dinucleotide, accompanied with the reduction of GSH. ${ }^{27} \mathrm{GSH} S$-transferase (GST) was estimated by measuring the rate of GSH conjugation with 1-chloro-2,4-dinitrobenzene as substrate. ${ }^{28}$

\section{Determination of GSH/GSSG in liver tissues}

The concentration of reduced GSH in liver tissues was measured following Ellman's method, ${ }^{29}$ whereas oxidized GSH was measured by the NADPH GR system at $412 \mathrm{~nm}$. The concentration was expressed as GSH disulfide (GSSG; $\mu \mathrm{M}$ ) in liver tissues. ${ }^{30}$ The GSH/GSSH ratio was then calculated by dividing the difference between the total GSH and GSSG concentrations.

\section{Determination of inflammatory markers}

The levels of the inflammatory cytokines (TNF $\alpha$ and IL-6) in serum were determined using commercially available enzyme-linked immunosorbent assay kits (eBioscience) according to the manufacturer's protocol. TNF $\alpha$ and IL-6 were determined from a standard curve, and the concentrations are expressed as $\mathrm{pg} / \mathrm{mL}$.

\section{Statistical analysis}

All results are presented as means \pm standard error of the mean. Statistical significance was assessed using one-way analysis of variance with post hoc pairwise comparisons between groups (groups I-VI) using the Bonferroni method. For all analyses, $P<0.05$ was considered to be significant, and $P<0.001$ was considered highly significant. Statistical analysis was performed using the computer statistical package SPSS 10.0 (SPSS Inc., Chicago, IL, USA).

\section{Results}

\section{Extraction and analysis}

The yield of AG diterpenoid lactone from A. paniculata dry leaves was $10.5 \mathrm{~g}$, which was $\sim 2.1 \%$ on a w/w basis. The melting point and retention-factor value for AG were 
recorded as $229^{\circ} \mathrm{C} \pm 1^{\circ} \mathrm{C}$ and 0.83 , respectively. AG was analyzed after purification for $\mathrm{C}, \mathrm{H}$, and $\mathrm{N}$ percentage composition. The carbon percentage in $\mathrm{AG}$ was recorded as $68.49 \%$ and that for hydrogen was $8.25 \%$, with nil recordings for the elemental nitrogen. The theoretical compositions for carbon and hydrogen in AG were C $68.57 \%$ and $\mathrm{H}$ 8.57\%, respectively. The comparative percentage-composition analysis indicated that AG, which was extracted and purified, was of sufficient purity, and could be used for further drug-like studies.

In FTIR scans, the characteristic AG lactone ring responded at $1,727 \mathrm{~cm}^{-1}$ and the conjugated $\mathrm{C}=\mathrm{C}$ vibrations were recorded at $1,674 \mathrm{~cm}^{-1}$, which matched well with a previous report. ${ }^{31}$ The $\mathrm{C}-\mathrm{O}$ stretching responses from the primary and the secondary alcoholic functions were recorded at $1,030 \mathrm{~cm}^{-1}$ and at $1,078 \mathrm{~cm}^{-1}$. AG $\mathrm{O}-\mathrm{H}$ stretching vibrations were recorded at $3,402 \mathrm{~cm}^{-1}$, indicating an intermolecular hydrogen-bonded structure, and the $\mathrm{O}-\mathrm{H}$ in-plane bending appeared at $1,420 \mathrm{~cm}^{-1}$ (Figure 1A). The UV $\lambda_{\text {max }}$ for AG in chloroform solution was recorded at $228 \mathrm{~nm}$ (Figure S1). Characteristic AG electrospray ionization mass spectrometry was recorded with $\mathrm{m} / \mathrm{z}: 373(\mathrm{M}+\mathrm{Na})^{+}$and $389(\mathrm{M}+\mathrm{K})^{+}$(Figure 1B). The results were typical and similar to an earlier report. ${ }^{32}$ AG was routinely analyzed by RP-HPLC, and the $\lambda_{\text {max }}$ was detected at $228 \mathrm{~nm}$, with retention time of 4.5 minutes.
A


B
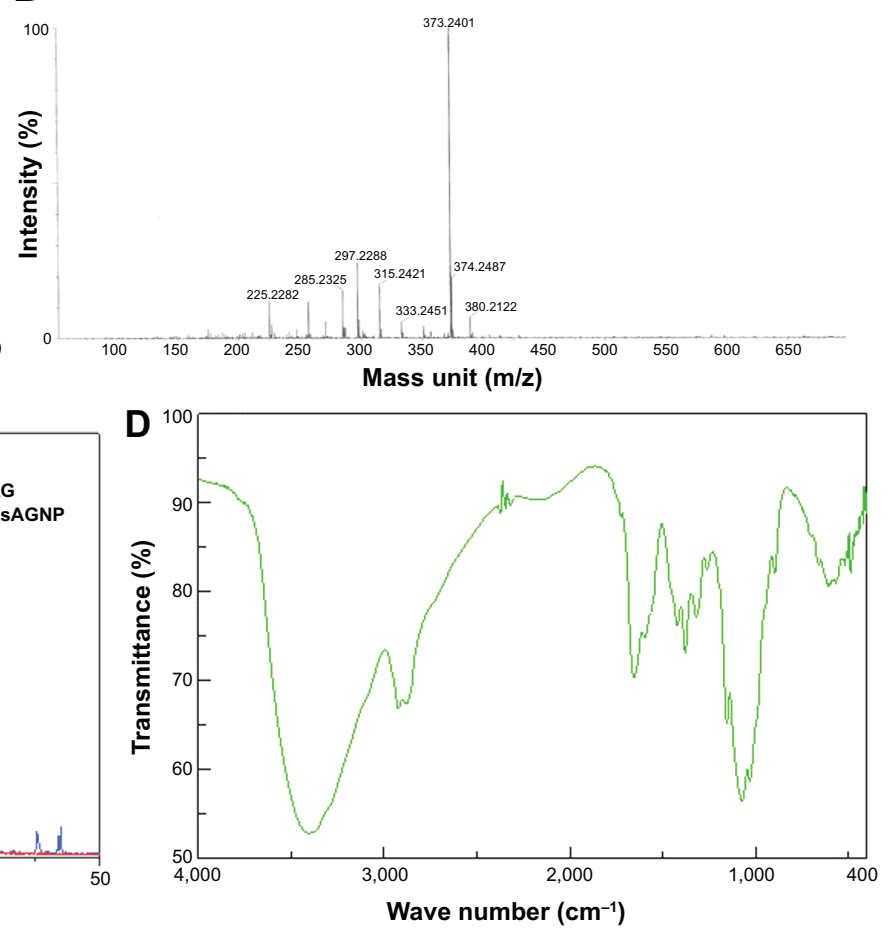



Figure I (A) FTIR scan of AG; (B) electrospray ionization mass spectra of AG; (C) X-ray diffraction spectra of AG and CsAGNP nanosystems; (D) FTIR scan of biopolymer Cs; (E) FTIR scan of CsAGNP nanosystem.

Abbreviations: Cs, chitosan; FTIR, Fourier-transform infrared; AG, andrographolide; CsAGNP, chitosan-modified AG nanoparticle. 
A standard curve was plotted from AG concentration versus peak area, and a linear relationship was established. ${ }^{18}$ The correlation coefficient $R^{2}$ was recorded using SigmaPlot 6.0 software as 0.9979 .

\section{Nanosystem characterization}

Average photon-correlation spectroscopy particle size of CsAGNPs was recorded as $229.7 \pm 17.17 \mathrm{~nm}$, with a nearGaussian particle distribution and polydispersity index of $0.234 \pm 0.02$ (Figure 2A). CsAGNPs exhibited cationic surface charge with a $\zeta$-potential value of $+34.4 \pm 1.87 \mathrm{mV}$. The AFM 3-D data for CsAGNP preparations (Figure 2B) matched with TEM observations (Figures S2 and S3) for mostly spherical particles with smooth surface topography and minimal coalescence. Entrapment efficiency is a crucial parameter governing the therapeutic performance of the delivery device. AG mass loading in CsAGNPs determined by RP-HPLC was $85.37 \% \pm 2.9 \%$.
XRD patterns of AG exhibited sharp peaks (Figure 1C) at a $2 \theta$ scattered angle $\left(2 \theta=10^{\circ}, 12^{\circ}, 15^{\circ}\right.$, and $\left.16^{\circ}\right)$, indicating its crystalline nature, similar to that in an earlier report. ${ }^{33}$ XRD study of CsAGNPs revealed no sharp characteristic peak of $\mathrm{AG}$, indicating that $\mathrm{AG}$ entrapment in the nanosystems likely occurred in amorphous form. A reaction in alizarin red was used for quantitative estimation of $\mathrm{Cs}$ in the final nanosystems. A standard graph plotted at $571 \mathrm{~nm}$ in an acidic $\mathrm{pH}$ environment ( $\mathrm{pH}$ 5) was applied, and the Cs mass-percentage entrapment on CsAGNPs was recorded from an average of four experiments as $74.02 \% \pm 3.78 \%$.

When scanned alone in FTIR spectroscopy (Figure 1D), Cs responded at $1,656 \mathrm{~cm}^{-1}$ and $1,591 \mathrm{~cm}^{-1}$ due to amide I and amide II vibrations, and the $-\mathrm{O}-\mathrm{H}$ response appeared at $3,404 \mathrm{~cm}^{-1}$. However, in CsAGNP FTIR scans, a strong shift for amide I appeared at $1,624 \mathrm{~cm}^{-1}$, likely due to electrostatic interaction alongside a weak signal for the
A

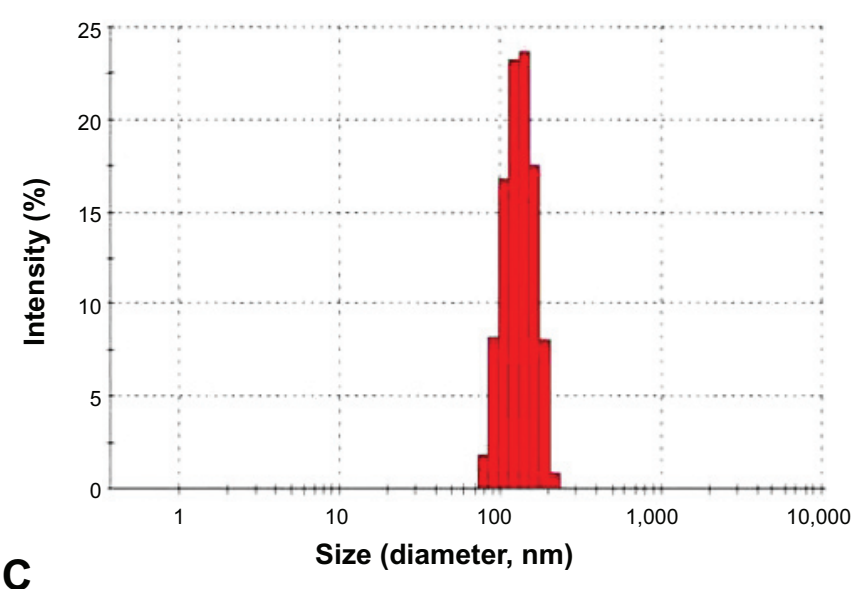

B

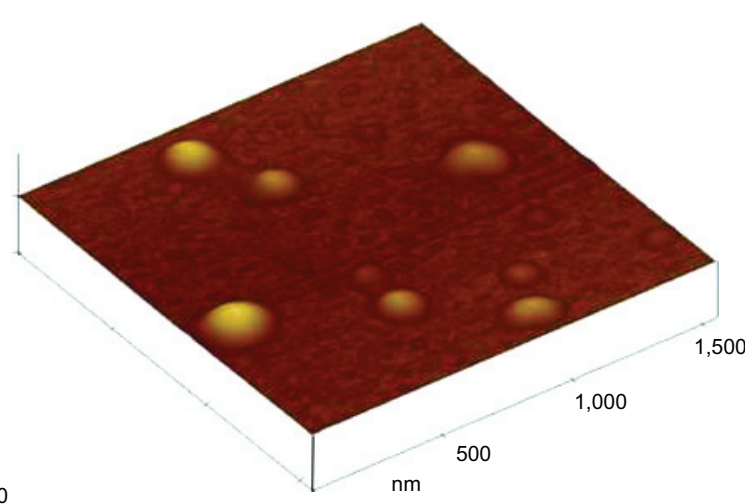



Figure 2 (A) Size distribution of CsAGNPs; (B) 3-D atomic force microscopy data of CsAGNPs; (C) In vitro dissolution studies for CsAGNPs (blue) in phosphate buffer, $\mathrm{pH}$ 7.4.

Abbreviations: AG, andrographolide; CsAGNPs, chitosan-modified AG nanoparticles. 
designated chitosan $-\mathrm{N}-\mathrm{H}$ vibrations (Figure 1E). Strong electrostatic interaction between biopolymeric $\mathrm{COO}^{-}$and Cs $\mathrm{NH}_{3}^{+}$functions assisted in Cs attachments on Pluronic stabilized PLGA NP surface. That was also one reason likely for positive $\zeta$-value observation due to Cs embedding in the case of CsAGNPs. When AG PLGA NPs alone were studied in photon correlation-spectroscopy, the $\zeta$-value observation and particle size recorded were $-29.7 \pm 1.41 \mathrm{mV}$ and $181 \pm 12.60 \mathrm{~nm}$, respectively.

\section{In vitro dissolution}

Time-dependent dissolution for CsAGNPs was studied, and the cumulative percentage release over time is plotted in Figure 2C. The release response for CsAGNP nanosystems showed a biphasic character. A rapid dissolution of AG occurred only up to 8 hours, followed by a sustained effect over an observation period of 432 hours. In CsAGNP dissolution for AG, the initial burst was comparatively slow, possibly due to a Cs and PLGA matrix biopolymer interaction surface. CsAGNPs showed almost a 50\% release of the therapeutic load by 132 hours, and $83 \%$ of the initial AG load was traced during the study period. Similar observations for sustained release in $\mathrm{Cs}$ bedecking were recorded earlier in Cs-embedded NPs for Sn-38. ${ }^{20}$

The drug-release data of CsAGNPs were further fitted to a power-law equation to account for the overlapping effects of Fickian diffusion and likely viscoelastic relaxation in polymeric carrier systems. ${ }^{34}$ CsAGNPs produced a release exponent (n) value of 0.52 , indicative of anomalous transport, ${ }^{35}$ wherein both diffusion and dissolution are quite indistinguishable and occur simultaneously. ${ }^{36}$

\section{Liver-function markers and histopathology}

Due to hepatic injury, the liver-function markers ALT, AST, and ALP were elevated significantly in the $\mathrm{CCl}_{4}$-treated group when compared to the control. AG and CsAGNPs attenuated the $\mathrm{CCl}_{4}$-induced elevation of serum enzyme parameters (Table 1, $P<0.001$ ). However, when the AG- and CsAGNP-treated groups were compared, the latter treatment group showed significant $(P<0.05)$ improvement in all parameters.

Histopathological observations of the liver tissues of the $\mathrm{CCl}_{4}$-treated groups (Figure 3) indicated disruptions in the liver cytoarchitecture. Hepatocellular necrotic lesions, leukocyte infiltration, disintegrated central veins, and fatty changes were observed in the $\mathrm{CCl}_{4}$-treated group. In case of group I, no necrotic lesions or vascular degradation was noted and the cellular architecture was normal (Figure 3). Animals pretreated with AG showed comparative improvements in histology, as evident from the mild necrotic lesions. The CsAGNP-treated group showed ameliorated $\mathrm{CCl}_{4}$-induced hepatic damage, and the liver tissues showed significant regeneration, as evident from the absence of cellular necrosis and inflammatory infiltrates, which was comparable to that in the control group.

\section{Hepatic lipid peroxidation}

$\mathrm{CCl}_{4}$ treatment significantly increased the level of hepatic lipid peroxidation, as judged by MDA levels of liver homogenate (Table 1). Both AG and CsAGNP treatment decreased hepatic MDA levels; however, the new nanosystem afforded an enhanced degree of protection $(34.4 \%$ decrease compared to the $\mathrm{CCl}_{4}$ group) than that for the AG-treatment group (27.1\% decrease compared to the $\mathrm{CCl}_{4}$ group), which was statistically significant $(P<0.05)$.

\section{Effect of CsAGNPs on hepatic antioxidant enzymes}

The activities of the antioxidant enzymes (SOD, CAT, GPx, GR, and GST) in the liver tissue are shown in Figure 4. The activity of antioxidant-enzyme activity was significantly $(P<0.001)$ decreased in the $\mathrm{CCl}_{4}$-treated group compared to the control group. AG- or CsAGNP-pretreated groups showed significant increase in activities of most of the antioxidant enzymes

Table I Liver function markers and lipid peroxidation in treatment conditions

\begin{tabular}{llll}
\hline Groups & AST (IU/L) & ALT (IU/L) & $\begin{array}{c}\text { ALP (IU/L) } \\
\text { (nM/mg liver tissue) }\end{array}$ \\
\hline Control & $48.33 \pm 5.44$ & $71.50 \pm 8.36$ & $66.00 \pm 8.39$ \\
CCl $_{4}$ & $157.83 \pm 19.77$ & $201.83 \pm 19.53$ & $187.83 \pm 17.16$ \\
AG & $106.67 \pm 13.4 I^{*}$ & $136.67 \pm 20.26 *$ & $140.00 \pm 16.20 *$ \\
CsAGNPs & $84.83 \pm 7.25 * * *$ & $105.50 \pm 10.60 *, * *$ & $86.83 \pm 10.17$ \\
\hline
\end{tabular}

Notes: $* P<0.001$, significant difference compared with $\mathrm{CCl}_{4}$-treated group; $* * P<0.05$, significant difference compared among AG- and CsAGNP-treated groups. Results are expressed as means \pm standard deviation $(n=6)$.

Abbreviations: AST, aspartate aminotransferase; ALT, alanine transaminase; ALP, alkaline phosphatase; MDA, malonyldialdehyde; AG, andrographolide; CsAGNPs, chitosan-modified AG nanoparticles. 

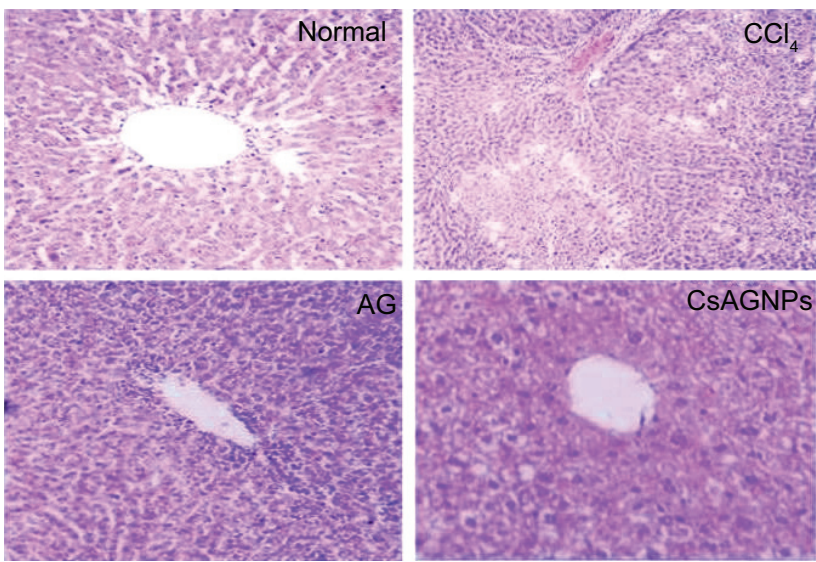

Figure 3 Histopathological changes of liver cytoarchitecture after various treatments, magnification $400 \times$, normal liver, $\mathrm{CCl}_{4}$-treated liver, AG-treated liver, CsAGNPtreated liver.

Abbreviations: AG, andrographolide; CsAGNPs, chitosan-modified AG nanoparticles.

$(P<0.05)$ when compared to that of the $\mathrm{CCl}_{4}$-treated group. However, CsAGNPs showed marked improvement (SOD $57.2 \%$, CAT $53.1 \%$, GPx 50.6\%, GR 30\%, and GST 50.6\%) when compared to that of the only AG-treated group (SOD $25.8 \%$, CAT $28 \%$, GPx $21 \%$, GR $13.5 \%$, and GST 20.5\%).

\section{Effect of CsAGNPs on hepatic nonenzymatic antioxidants}

Mice treated with $\mathrm{CCl}_{4}$ had a marked depletion $(P<0.001)$ in hepatic GSH levels with a concomitant increase in GSSG levels $(P<0.001)$ in comparison with the control group. That impacted the GSH/GSSG ratio in the liver tissues (Table 2). Administration of $\mathrm{AG}$ resulted in moderate restoration of hepatic GSH content (35\%), which was statistically insignificant; however treatment with CsAGNPs markedly improved hepatic GSH content (62.4\%, $P<0.05)$. Concomitantly, the only AG treatment failed to reduce hepatic GSSG levels significantly $(13 \%, P<0.4)$, but CsAGNP treatment resulted in a significant decrease in hepatic GSSG levels (22.2\%, $P=0.02$ ). A similar observation was reflected in the GSH/GSSG ratio, where CsAGNP treatment in significant improvement $(111.9 \%, P<0.05)$, whereas the only AG treatment failed to elicit such response $(56.3 \%, P>0.05)$.

\section{Effect on serum levels of inflammatory markers}

$\mathrm{CCl}_{4}$ treatment caused a significant increase in the levels of both the inflammatory markers TNF $\alpha$ and IL-6 in serum samples $(P<0.001)$. Pretreatment with AG and CsAGNPs helped in lowering the TNF $\alpha$ and IL-6 levels (Table 3) when compared to the $\mathrm{CCl}_{4}$-treated control group. However, a superior impact in TNF $\alpha$ levels in the CsAGNP-treated group was recorded and statistically significant when compared to that of the only AG-treated group $(P<0.05)$.

\section{Discussion}

AG is a potent anti-inflammatory, antiapoptotic, and immunomodulator compound, but is imperfect in therapeutics due to inadequate drug pharmacokinetics. A. paniculata extracts are traditionally applied for multiple ailments, but seasonal variation in compound yield is one of the known limiting factors in intended pharmacology efficacy. ${ }^{37}$ Structural tailoring attempted earlier on AG diterpenoid lactone was not very successful in activity enhancement or pharmacokinetics. ${ }^{38}$ It is also imperative to follow an appropriate isolation and standardization technique for optimum product yield. A further dissolution enhancement is also crucial in order to meet the requirements in drug-like applications of AG. Leaves collected during the month of September provided a higher yield of nearly $2 \% \mathrm{w} / \mathrm{w}$ in a solvent-extraction and chromatography procedure. New nanosystems with AG as a payload were prepared exploiting the similar solubility profile of AG and the biopolymer PLGA. Cs bedecking on the PLGA NP surface was considered for an improvement in biocompatibility and permeability necessary for peroral activity. Besides, the Cs polysaccharide has a number of $-\mathrm{OH}$ and $-\mathrm{NH}$ groups that provide ample opportunities for intermolecular hydrogen bonding with PLGA, which allows favorable particle engineering for the newer nanosystems. An FTIR study in $\mathrm{KBr}$ pellets has amply demonstrated structurally favorable biopolymer interactions (Figure 1E). Bioconjugation of Cs due to noncovalent interactions on the NPs provided for a $\zeta$-shift and biopolymer bedecking. AG dissolution in the case of CsAGNPs was also consistent and traceable following an anomalous mass transport.

Liver is a self-regenerating tissue, but progressive and often irreversible degenerations are commonly caused by different chemicals, drugs, virus overloads, and in cases of alcoholic and nonalcoholic fatty liver diseases. Marked increases in liver diseases due to lifestyle changes and toxic exposures are presently one alarming concern in personal health management. ${ }^{39,40}$ Progressive oxidative stress and persistent inflammatory conditions are some of the paramount causes for critical hepatotoxicity. ${ }^{41}$ Considerable research for hepatoprotective therapeutics is under way, and liver injury by $\mathrm{CCl}_{4}$ intoxication serves as one of the most well-characterized in vivo models for investigation of these newer entities. The $\mathrm{CCl}_{4}$ chemical hepatotoxin induces free radical-mediated hepatocellular damages that eventually lead 



Figure 4 Levels of hepatic enzymatic antioxidant markers before and after $\mathrm{CCl}_{4}$-induced hepatotoxicity. Results are expressed as means \pm standard deviation ( $\mathrm{n}=6$ ). Notes: ${ }^{* P}<0.00 I$, significant difference compared with $\mathrm{CCl}_{4}$-treated group; ${ }^{*} * P<0.05$ significant difference compared among AG- and CsAGNP-treated groups.

Abbreviations: AG, andrographolide; CsAGNPs, chitosan-modified AG nanoparticles; SOD, superoxide dismutase; CAT, catalase; GPx, glutathione peroxidase; GR, glutathione reductase; GST, glutathione S-transferase.

to membrane-lipid peroxidation and necrosis. $\mathrm{CCl}_{4}$ toxicity correlates well with the conditions of oxidative stress in the decrease of antioxidant enzymatic activity and progressive loss of nonenzymatic antioxidants. ${ }^{42}$ Such enzymes as SOD, CAT, GR, and GPx are all downregulated in $\mathrm{CCl}_{4}$-induced hepatotoxicity. $\mathrm{CCl}_{4}$ is also known to decrease the detoxification system produced by GST. ${ }^{43}$ CsAGNPs afforded an increase in the activities of the antioxidant enzymes in $\mathrm{CCl}_{4}$-damaged liver. This effect is a contrastingly significant improvement over that of AG treatment alone (Figure 4). $\mathrm{CCl}_{4}$ also causes elevation of marker enzymes, such as ALT and AST, in serum, due to hepatocellular damage and release of cytoplasmic enzymes into the bloodstream. ${ }^{43}$ Once exposed to $\mathrm{CCl}_{4}$ hepatocellular damage, the membrane permeability gets altered with the release of cytoplasmic enzymes into the bloodstream.

In our study, a significant increment of ALT (64\%) and AST (64\%) occurred after only $\mathrm{CCl}_{4}$ intoxication, indicating 
Table 2 Nonenzymatic hepatic antioxidants in $\mathrm{CCl}_{4}$-induced hepatotoxicity

\begin{tabular}{llll}
\hline Groups & $\begin{array}{l}\text { Hepatic GSH } \\
(\mu \mathrm{M} / \mathbf{m g} \text { liver tissue })\end{array}$ & $\begin{array}{l}\text { Hepatic GSSG } \\
(\mu \mathrm{M} / \mathrm{mg} \text { liver tissue })\end{array}$ & GSH/GSSG \\
\hline Control & $8.618 \pm 1.20$ & $0.755 \pm 0.09$ & $11.66 \pm 2.86$ \\
CCl $_{4}$ & $4.567 \pm 0.66$ & $1.082 \pm 0.15$ & $4.28 \pm 0.82$ \\
AG & $6.17 \pm 1.21$ & $0.943 \pm 0.12$ & $6.69 \pm 1.81$ \\
CsAGNPs & $7.417 \pm 1.33^{*}$ & $0.843 \pm 0.13^{*}$ & $9.07 \pm 2.72^{*}$ \\
\hline
\end{tabular}

Notes: $* P<0.05$, significant difference compared with $\mathrm{CCl}_{4}$-treated group. Results expressed as means \pm standard deviation ( $\mathrm{n}=6$ ).

Abbreviations: GSH, glutathione; GSSG, GSH disulfide; AG, andrographolide; CsAGNPs, chitosan-modified AG nanoparticles.

loss of functional integrity of the liver-cell membranes. It was observed further that among the AG- or CsAGNP-pretreatment groups, CsAGNPs favored enhanced suppression of $\mathrm{CCl}_{4}$-induced liver injury, which was evident in a decrease of serum marker enzymes. Similarly, suppression of elevated ALP activities by the nanosystems indicated improvements in hepatobiliary functions. Histopathological examination of hepatocellular damage after $\mathrm{CCl}_{4}$ administration indicated typical centrilobular necrosis, ballooning of hepatocytes, infiltration, and steatosis. CsAGNP nanosystems maintained normal histoarchitecture and demonstrated a regenerative effect on the hepatocytes even after oxidative stress induced by $\mathrm{CCl}_{4}$ administration (Figure 3). AG to some extent ameliorated degenerative changes, but some of the hepatocytes still demonstrated restricted ballooning necrotic changes. CsAGNPs were therefore demonstrably effective in exerting hepatoprotective efficacies.

One likely reason for a verifiable efficacy of CsAGNPs was considered due to liver-centric dissolution. NPs, particularly the cationic ones with a diameter of $\sim 200 \mathrm{~nm}$, are prone to endocytosis and rapid localization in the liver tissues. ${ }^{44} \mathrm{In}$ order to understand such effects, a group of 12 animals were fed separately with a similar Cs-engineered PLGA nanosystem carrying fluorescein isothiocyanate dye in place of AG. Liver-histology study under confocal microscopy was then performed at hourly intervals (Figure 5). The observations clearly demonstrated a rapid rise in FITC fluorescein isothiocyanate, which reached saturation within 4 hours of

Table 3 Serum levels of inflammatory markers

\begin{tabular}{lll}
\hline Groups & $\begin{array}{l}\text { Serum TNF } \alpha \text { level } \\
(\mathbf{p g} / \mathbf{m L})\end{array}$ & $\begin{array}{l}\text { Serum IL-6 level } \\
(\mathbf{p g} / \mathbf{m L})\end{array}$ \\
\hline Control & $77.50 \pm 9.20$ & $52.8 \pm 6.09$ \\
$\mathrm{CCl}_{4}$ & $247.82 \pm 20.66$ & $109.33 \pm 11.15$ \\
$\mathrm{AG}$ & $149.13 \pm 16.26^{*}$ & $71.94 \pm 8.12^{*}$ \\
CsAGNPs & $112.18 \pm 12.52^{* * * *}$ & $63.84 \pm 6.13^{*}$ \\
\hline
\end{tabular}

Notes: $* P<0.001$, significant difference compared with $\mathrm{CCl}_{4}$-treated group; $* * P<0.05$, significant difference compared among AG- and CsAGNP-treated groups. Results expressed as means \pm standard deviation $(n=6)$.

Abbreviations: AG, andrographolide; CsAGNPs, chitosan-modified AG nanoparticles. administration. CsAGNP hydrodynamic size observed was $229.7 \pm 17.17 \mathrm{~nm}$, and the particles were carrying a surface charge of $+34.4 \pm 1.87 \mathrm{mV}$, which could be some of the basis for enhanced hepatoprotection extended by the new nanosystems.

In $\mathrm{CCl}_{4}$-induced hepatic injury, free radicals activate Kupffer cells, which mediate the hepatic inflammation process by producing TNF $\alpha$ and other proinflammatory cytokines like IL-6. Elevation of IL- 6 and TNF $\alpha$ levels are always associated in liver-damage conditions. ${ }^{45}$ Proinflammatory cytokines are known further to induce apoptosis and cellular necrosis. Moreover, elevated levels of cytokines induce nitric oxide production, a highly reactive oxidant molecule, leading to an imbalance in the oxidative state. In our study, the serum levels of TNF $\alpha$ were low in the normal control animals, but increased considerably in the $\mathrm{CCl}_{4}$-treated animals. CsAGNP pretreatment strongly inhibited the elevated TNF $\alpha$ production after $\mathrm{CCl}_{4}$ injection. Reduced levels of TNF $\alpha$ might play a protective role against liver damage by inducing hepatocyte proliferation and by modulation of the release of tissue-repair mediators.

MDA is the indicator of lipid peroxidation. MDA levels increased upon $\mathrm{CCl}_{4}$ administration, suggesting increased lipid-peroxidation activities due to the formation
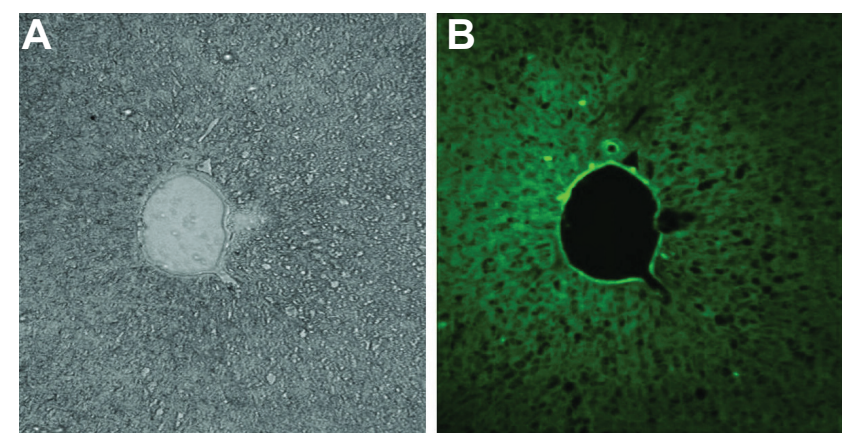

Figure 5 Fluorescein isothiocyanate-labeled nanoparticle localization in the liver after 4 hours of administration.

Notes: (A) Transmitted image; (B) fluorescent image. 
of excessive ROS and failure of the antioxidant defense mechanism, which led to tissue damage. MDA levels were restored toward normal after AG and CsAGNP treatment, with a better restorative effect being observed in the case of the CsAGNP-treated group. GSH is one of the most abundant low-molecular-weight thiols, and protects cells against ROS as well as toxic compounds by its reduction ability. The level of GSH gives a clear indication of the cellular oxidation state. GSH plays a key role in eliminating the reactive toxic metabolites of $\mathrm{CCl}_{4}$, and depletion of GSH stores leads to cellular necrosis. GPx-mediated reduction of oxygen radicals converts GSH to its oxidized form - GSSG. GR, an NADPH-dependent enzyme, converts GSSG to GSH. GSH/GSSG is the major redox couple in animal cells, and in normal conditions, the cellular GSSG level remains in a very low concentration, around $2 \%-10 \%$ of total GSH level. ${ }^{46}$ In an oxidative environment, a considerable shift toward GSSG was observed, and as a result the GSH/GSSG ratio decreased, indicating a state of oxidative stress. ${ }^{47}$ The GSH/GSSG ratio was increased due to both the AG and CsAGNP treatments. However, the effect of CsAGNP was overriding.

\section{Conclusion}

We summarize that the engineered AG nanosystem CsAGNPs extended a tissue-centric defense against oxidative stress-induced liver-damage conditions. Upregulation of various antioxidant enzymatic machineries and restoration of hepatic GSH levels are some of the reasons identified for superior hepatoprotection. The AG nanosystem directed a favorable cytokine regulation and facilitated recovery in $\mathrm{CCl}_{4}$-damaged hepatoarchitecture. Confocal microscopy observations recorded a rapid localization of CsAGNPs in the liver tissue within 4 hours of administration via the oral route. New engineered AG nanosystems therefore extend one possibility in cases of oxidative stress and hepatocellular inflammatory conditions. Further pharmacokinetic studies of similar engineered nanosystems are warranted to permit entry in clinical studies, and such study is under way.

\section{Acknowledgments}

A Research Associateship to Dr Partha Roy and Senior Research Fellowship to Suvadra Das from the Council of Scientific and Industrial Research and DST PURSE are gratefully acknowledged. Dr Runa Ghosh Auddy would like to thank CRNN, University of Calcutta for a Research Associateship. Our group is thankful to TEQIP for financial assistance and CRNN, University of Calcutta for extending the research facilities related to the work.

\section{Disclosure}

The authors report no conflicts of interest in this work.

\section{References}

1. American Liver Foundation. Liver Wellness: Increasing Public Awareness of Liver Health - An Introduction to Liver Care. New York: American Liver Foundation; 2009. Available from: www.liverfoundation.org/downloads/alf_download_29.pdf. Accessed August 20, 2013.

2. Mishra N, Yadav NP, Rai VK, et al. Efficient hepatic delivery of drugs: novel strategies and their significance. Biomed Res Int. 2013; 2013:382184.

3. Henninger C, Huelsenbeck J, Huelsenbeck S, et al. The lipid lowering drug lovastatin protects against doxorubicin-induced hepatotoxicity. Toxicol Appl Pharmacol. 2012;261(1):66-73.

4. Vidal F, Gutiérrez F, Gutiérrez M, et al. Pharmacogenetics of adverse effects due to antiretroviral drugs. AIDS Rev. 2010;12(1):15-30.

5. Nahin RL, Straus SE. Research into complementary and alternative medicine: problems and potential. BMJ. 2001;322(7279):161-164.

6. Chen $\mathrm{HW}$, Lin $\mathrm{AH}$, Chu $\mathrm{HC}$, et al. Inhibition of TNF- $\alpha$-induced inflammation by andrographolide via down-regulation of the PI3K/ Akt signaling pathway. J Nat Prod. 2011;74(11):2408-2413.

7. Mishra SK, Sangwan NS, Sangwan RS. Andrographis paniculata (kalmegh): a review. Pharmacogn Rev. 2007;1(2):283-298.

8. Subramanian R, Asmawi MZ, Sadikun A. A bitter plant with a sweet future? A comprehensive review of an oriental medicinal plant: Andrographis paniculata. Phytochem Rev. 2012;11(1):39-75.

9. Niranjan A, Tewari SK, Lehri A. Biological activities of kalmegh (Andrographis paniculata Nees) and its active principles - a review. Indian J Nat Prod Resour. 2010;1(2):125-135.

10. Lala S, Nandy AK, Mahato SB, Basu MK. Delivery in vivo of 14-deoxy11-oxoandrographolide, an antileishmanial agent, by different drug carriers. Indian J Biochem Biophys. 2003;40(3):169-174.

11. Zhu SP, Kang BA. [Distribution and excretion of [35S] $\mathrm{NaHSO}_{3}-$ andrographolide by autoradiography (author's transl)]. Zhongguo Yao Li Xue Bao. 1981;2(4):266-269. Chinese.

12. Ye L, Wang T, Tang L, et al. Poor oral bioavailability of a promising anticancer agent andrographolide is due to extensive metabolism and efflux by P-glycoprotein. J Pharm Sci. 2011;100(11):5007-5017.

13. Tang C, Gu G, Wang B, et al. Design, synthesis and biological evaluation of andrographolide derivatives as potent hepatoprotective agents. Chem Biol Drug Des. 2014;83(3):324-333.

14. Roy P, Das S, Auddy RG, Saha A, Mukherjee A. Engineered andrographolide nanoparticles mitigate paracetamol hepatotoxicity in mice. Pharm Res. 2013;30(5):1252-1262.

15. Yang T, Sheng HH, Feng NP, Wei H, Wang ZT, Wang CH. Preparation of andrographolide-loaded solid lipid nanoparticles and their in vitro and in vivo evaluations: characteristics, release, absorption, transports, pharmacokinetics, and antihyperlipidemic activity. J Pharm Sci. 2013; 102(12):4414-4425.

16. Wong FW, Chan W, Lee SS. Resistance to carbon tetrachloride-induced hepatotoxicity in mice which lack CYP2E1 expression. Toxicol Appl Pharmacol. 1988;153(1):109-118.

17. Ichinose T, Miller MG, Shibamoto T. Determination of free malonaldehyde formed in liver microsomes upon $\mathrm{CCl}_{4}$ oxidation. $J \mathrm{Appl}$ Toxicol. 1994;14(6):453-455.

18. Roy P, Das S, Bera T, Mondol S, Mukherjee A. Andrographolide nanoparticles in leishmaniasis: characterization and in vitro evaluations. Int J Nanomedicine. 2010;5:1113-1121.

19. Roy P, Das S, Mondal A, Chatterji U, Mukherjee A. Nanoparticle engineering enhances anticancer efficacy of andrographolide in MCF-7 cells and mice bearing EAC. Curr Pharm Biotechnol. 2012;13(15):2669-2681. 
20. Guo M, Rong WT, Hou J, et al. Mechanisms of chitosan-coated poly(lactic-co-glycolic acid) nanoparticles for improving oral absorption of 7-ethyl-10-hydroxycamptothecin. Nanotechnology. 2013;24(24):245101.

21. Upur H, Amat N, Blazekovic B, Talip A. Protective effect of Cichorium glandulosum root extract on carbon tetrachloride-induced and galactosamine-induced hepatotoxicity in mice. Food Chem Toxicol. 2009;47(8):2022-2030.

22. Yousef MI, Omar SA, El-Guendi MI, Abdelmegid LA. Potential protective effects of quercetin and curcumin on paracetamol-induced histological changes, oxidative stress, impaired liver and kidney functions and haematotoxicity in rat. Food Chem Toxicol. 2010;48(11):3246-3261.

23. Ohkawa H, Ohishi N, Yagi K. Assay for lipid peroxides in animal tissues by thiobarbituric acid reaction. Anal Biochem. 1979;95(2):351-380.

24. Misra HP, Fridovich I. The generation of superoxide radical during the autoxidation of hemoglobin. J Biol Chem. 1972;247(21):6960-6962.

25. Best TM, Fiebig R, Corr DT, Brickson S, Ji L. Free radical activity, antioxidant enzyme, and glutathione changes with muscle stretch injury in rabbits. J Appl Physiol. 1999;87(1):74-82.

26. Czuczejko J, Bronisław A, Zachara BA, Staubach-Topczewska E, Halota W, Kędziora J. Selenium, glutathione and glutathione peroxidases in blood of patients with chronic liver diseases. Acta Biochim Pol. 2003;50(4):1147-1154.

27. Beutler E. Effect of flavin compounds on glutathione reductase activity: in vivo and in vitro studies. J Clin Invest. 1969;48(10):1957-1966.

28. Habig WH, Pablst MJ, Jakoby WB. Glutathione-S-transferase. The first enzymatic step in mercapturic formation. J Biol Chem. 1974; 249(22):7130-7139.

29. Ellman GL. Tissue sulfhydryl groups. Arch Biochem Biophys. 1959; 82(1):70-77.

30. Cheng CF, Pan TM. Protective effect of Monascus-fermented red mold rice against alcoholic liver disease by attenuating oxidative stress and inflammatory response. J Agric Food Chem. 2011;59(18): 9950-9957.

31. Cava MP, Chan WR, Haynes LJ, Johnson LF, Weinstein B. The structure of andrographolide. Tetrahedron. 1962;18(4):397-403.

32. Qizhen D, Jerz G, Winterhalter P. Separation of andrographolide and neoandrographolide from the leaves of Andrographis paniculata using high-speed counter-current chromatography. J Chromatogr A. 2003;984(1):147-151.

33. Imsanguan P, Pongamphai S, Douglas S, Teppaitoon W, Douglas PL. Supercritical antisolvent precipitation of andrographolide from Andrographis paniculata extracts: effect of pressure, temperature and $\mathrm{CO}_{2}$ flow rate. Powder Technol. 2010;200(3):246-253.
34. Brazel CS, Peppas NA. Modeling of drug release from swellable polymers. Eur J Pharm Biopharm. 2000;49(1):47-58.

35. Siepmann J, Siepmann F. Mathematical modeling of drug delivery. Int J Pharm. 2008;364(2):328-343.

36. Arifin DY, Lee LY, Wang CH. Mathematical modeling and simulation of drug release from microspheres: implications to drug delivery systems. Adv Drug Deliv Rev. 2006;58(12-13):1274-1325.

37. Sharma SN, Sahu S, Jha Z, Sharma DK. Evaluation of seasonal variation in relation to secondary metabolite and biomass production of Andrographis paniculata. J Nat Remedies. 2012;12(1):39-46.

38. Nanduri S, Nyavanandi VK, Thunuguntla SS, et al. Synthesis and structure-activity relationships of andrographolide analogues as novel cytotoxic agents. Bioorg Med Chem Lett. 2004;14(18):4711-4717.

39. Blachier M, Leleu H, Peck-Radosavljevic M, Valla DC, RoudotThoraval F. The burden of liver disease in Europe: a review of available epidemiological data. J Hepatol. 2013;58(3):593-608.

40. Asrani SK, Larson JJ, Yawn B, Therneau TM, Kim WR. Underestimation of liver-related mortality in the United States. Gastroenterology. 2013;145(2):375-382.

41. Reyes-Gordillo K, Segovia J, Shibayama M, Vergara P, Moreno MG, Muriel P. Curcumin protects against acute liver damage in the rat by inhibiting NF- $\kappa \mathrm{B}$, proinflammatory cytokines production and oxidative stress. Biochim Biophys Acta. 2007;1770(6):989-996.

42. Yu WG, Qian J, Lu YH. Hepatoprotective effects of $2^{\prime}, 4^{\prime}$-dihydroxy6'-methoxy-3',5'-dimethylchalcone on $\mathrm{CCl}_{4}$-Induced acute liver injury in mice. J Agric Food Chem. 2011;59(24):12821-12829.

43. Raj S, Gothandam KM. Hepatoprotective effect of polyphenols rich methanolic extract of Amorphophallus commutatus var. wayanadensis against $\mathrm{CCl}_{4}$ induced hepatic injury in swiss albino mice. Food Chem Toxicol. 2014;67:105-112.

44. Desai N. Challenges in development of nanoparticle-based therapeutics. AAPS J. 2012;14(2):282-295.

45. Das S, Santra A, Lahiri S, Guha Mazumder DN. Implications of oxidative stress and hepatic cytokine (TNF- $\alpha$ and IL-6) response in the pathogenesis of hepatic collagenesis in chronic arsenic toxicity. Toxicol Appl Pharmacol. 2005;204(1):18-26.

46. Wu G, Fang YZ, Yang S, Lupton JR, Turner ND. Glutathione metabolism and its implications for health. J Nutr. 2004;134(3):489-492.

47. Das SK, Vasudevan DM. Modulation of lecithin activity by vitamin B complex to treat long term consumption of ethanol induced oxidative stress in liver. Indian J Exp Biol. 2006;44(10):791-801. 


\section{Supplementary materials}
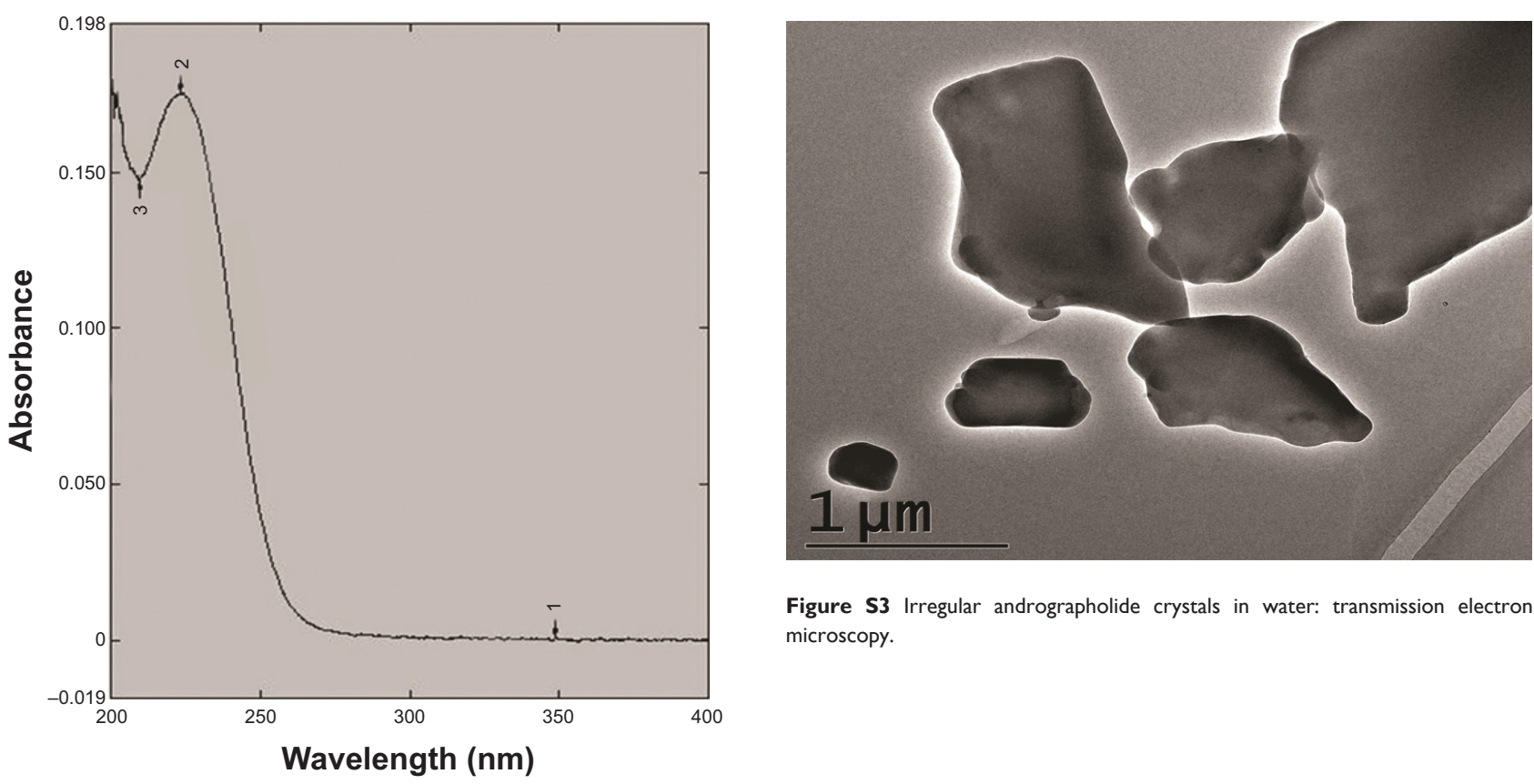

Figure S3 Irregular andrographolide crystals in water: transmission electron microscopy.

Figure SI Ultraviolet maximum absorbance for andrographolide extracted in $5 \mu \mathrm{g} / \mathrm{mL}$ chloroform solution.

Note: The arrows (numbered I, 2, and 3), indicate specific peak positions for andrographolide in UV-visible scanning.

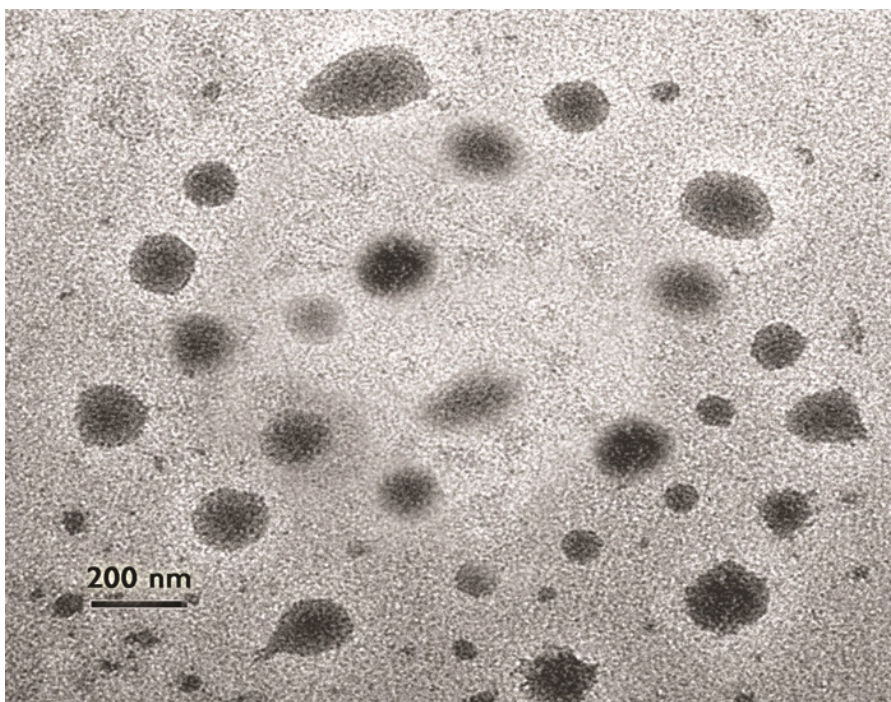

Figure S2 Engineered andrographolide nanosystems in water: transmission electron microscopy.

International Journal of Nanomedicine

\section{Publish your work in this journal}

The International Journal of Nanomedicine is an international, peerreviewed journal focusing on the application of nanotechnology in diagnostics, therapeutics, and drug delivery systems throughou the biomedical field. This journal is indexed on PubMed Central,

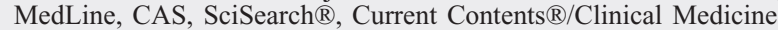

\section{Dovepress}

Journal Citation Reports/Science Edition, EMBase, Scopus and the Elsevier Bibliographic databases. The manuscript management system is completely online and includes a very quick and fair peer-review system, which is all easy to use. Visit http://www.dovepress.com/ testimonials.php to read real quotes from published authors.

Submit your manuscript here: http://www.dovepress.com/international-journal-of-nanomedicine-journal 